Conclusions TB screening in HIV-1 patients by serial OFT-GIT assays may be of clinical value due to its excellent reproducibility. However, OFT-GIT conversions and reversions should be interpreted carefully as their clinical significance remains to be established.

\section{P2.046 EVALUATION OF MATRIX-ASSISTED LASER DESORPTION/ IONIZATION TIME-OF-FLIGHT MASS SPECTROMETRY (MALDI-TOF-MS) FOR SPECIES IDENTIFICATION WITHIN THE NEISSERIA GENUS - EFFECTIVE ALTERNATIVE TO CONVENTIONAL TECHNIQUES?}

doi:10.1136/sextrans-2013-051184.0311

'D Golparian, ${ }^{2 B}$ Dauphin, 'B Hellmark, ${ }^{3 B}$ Claesson, 'M Unemo. 'WHO Collaborating Centre for Gonorrhoea and other STIs, Department of Laboratory Medicine, Microbiology, Örebro University Hospital, ÖREBRO, Sweden; ${ }^{2}$ Assistance Publique-Hôpitaux de Paris, Paris, France; ${ }^{3}$ Department of Clinical Microbiology, Skaraborg Hospital, Capio Diagnostic AB, Skövde, Sweden

Background Highly specific and sensitive discrimination between closely related pathogenic and commensal Neisseria spp is crucial because these species frequently colonise the same anatomical sites. Herein, two commercially available Matrix-Assisted Laser Desorption/Ionization Time-of-Flight Mass Spectrometry (MALDI-TOFMS) platforms and one independent software and database (Andromas) was compared to conventional phenotypic and genetic tests routinely used for identification of Neisseria spp.

Methods The performance of each platform, analysing 129 pathogenic isolates (Neisseria gonorrhoeae and Neisseria meningitidis) and 69 commensal Neisseria isolates (15 different species), was determined by deposition of one single culture colony to the MALDI plate and analysed in Microflex (Bruker, Germany) and VITEK MS (bioMérieux, France) according to the manufacturer's instructions. Subsequently, the acquired data from Microflex was submitted for analysis in the Andromas software and database (Andromas, France), which uses a different algorithm for species identification. Unfortunately, VITEK MS data is not compatible with Andromas. Phenotypic and genetic (16S rRNA gene sequencing) methods were used for final discrepancy analysis (still pending).

Results Microflex correctly identified all (100\%) N. gonorrhoeae and $\mathrm{N}$. meningitidis, however, four commensal isolates were indicated as possible N. meningitidis. Three of these four isolates were $\mathrm{N}$. kochii. The VITEK MS misidentified $1 \mathrm{~N}$. gonorrhoeae, $1 \mathrm{~N}$. meningitidis and 2 commensal isolates (both $\mathrm{N}$. kochii) were indicated as possible N. meningitidis. Finally, Microflex data analysed in the Andromas software and database correctly identified all (100\%) pathogenic and commensal strains.

Conclusion This study shows that both Microflex and VITEK MS discriminate pathogenic Neisseria species from commensal Neisseria species with a high, but not ideal, specificity. Furthermore, an optimal MALDI-TOF-MS platform should be compatible with secondary softwares and databases for confirmation. Importantly, the Microflex results analysed in the secondary software and database Andromas correctly identified all (100\%) pathogenic and commensal strains.

\section{P2.047 EVALUATION OF A MULTIPLEX REAL-TIME PCR ASSAY FOR RAPID DETECTION OF C.TRACHOMATIS AND $N$. GONORRHOEAE FROM GENITAL CLINICAL SPECIMENS}

doi:10.1136/sextrans-2013-051184.0312

'M Screm, 'M Di Santolo, 'C Scarparo, ${ }^{1,2} \mathrm{~A}$ Arzese. 'Microbiologia, Azienda Ospedaliero-Universitaria, Udine, Italy; ${ }^{2}$ Department of Experimental Clinical Medicine, University of Udine, Udine, Italy

Background Sexually transmitted diseases (STDs) are among the most common causes of illness in the world, being associated to acute disease, infertility, long term disability and death. In particular the prevalence of C.trachomatis and $N$. gonorrhoeae infections appear to remain steady or increasing, although both diagnostic and therapeuthical tools are available, at least in developed countries. Standard diagnostic protocols can be complex, time-consuming, and sensitivity of culture methods affected by specimen characteristics. In addition, certified methods are today required, especially in the management of cases of sexual abuse.

Methods Multiplex real-time PCR method (Xpert CT/NG, Cepheid) has been tested versus $N$. gonorrhoeae standard culture and C. trachomatis molecular assay (Artus C. trachomatis TM PCR Kit, Qiagen). 45 clinical samples (female and male urogenital swabs, ocular infections, first void of urine, seminal fluid, and external quality control samples - UK NEOAS) were selected and stored at $20^{\circ} \mathrm{C}$. Xpert CT/NG allowed to perform in one-step extraction, amplification and detection of C.trachomatis and N. gonorrhoeae DNA directly from all the variety of selected clinical specimens, within $60 \mathrm{~min}$

Results $100 \%$ results agreement was found between Xpert CT/NG and standard protocols, including expected results by UK-NEOAS quality assessment specimens. However, batch PCR is a multi-step time-consuming process, the turn-around-time (TAT) required from 8 to $48 \mathrm{hrs}$ versus the single technical and computer-assisted interpretation step by Xpert CT/NG, resulting in a $<$ two hrs TAT.

Conclusions Xpert CT/NG test is a easy, rapid, accurate and certified method in routine diagnostics of $N$. gonorrhoeae and C. trachomatis infections.

\section{P2.048 EVALUATION OF TWO COMMERCIAL REAL-TIME PCR ASSAYS FOR DETECTION OF MYCOPLASMA GENITALIUM IN UROGENITAL SPECIMENS}

doi:10.1136/sextrans-2013-051184.0313

${ }^{1,2} \mathrm{C}$ Le Roy, ${ }^{1,2,3}$ S Pereyre, ${ }^{1,2,3}$ C Bébéar. 'University of Bordeaux, Bordeaux, France; 2Institut National de la Recherche Agronomique, Bordeaux, France, ${ }^{3}$ Centre Hospitalier Universitaire de Bordeaux, Bordeaux, France

Objectives Mycoplama genitalium is a sexually transmitted organism associated with non-gonococcal urethritis in men and several inflammatory reproductive tract syndromes in women. Nucleic acid amplification tests are currently the only available methods for detection. The first commercially available real-time (RT-) PCR kits have been recently developed. We compared the TIB MOLBIOL LightMix $^{\circledR}$ Kit Mycoplasma genitalium and the Diagenode Mycoplasma genitalium real-time PCR kit to the in-house TaqMan RTPCR used routinely for the M. genitalium diagnostic.

Methods DNA extracts from $50 \mathrm{M}$. genitalium-negative and $53 \mathrm{M}$ genitalium-positive urogenital specimens collected between January 2010 and May 2011 at the Bordeaux University hospital, France, were retrospectively and systematically selected and thawed. DNA had been extracted using the MagNA Pure DNA isolation kit I (Roche Diagnostics). DNA extracts were evaluated by the TIBMOLBIOL LightMix ${ }^{\circledR}$ Kit Mycoplasma genitalium and the Diagenode Mycoplasma genitalium real-time PCR kit (DIA-MG-050 vs2) in comparison with a M. genitalium in-house RT-PCR targeting the $\mathrm{MgPa}$ adhesin gene using the cobas z480 analyser (Roche Diagnostics)

Results The in-house PCR was first evaluated using two thermal cyclers, LC480 and cobas z480 (Roche Diagnostics). As no significant difference was noted, the cobas z480 was used in the rest of the study. The clinical sensitivity was $98 \%, 92 \%$ and $100 \%$ for the LightMix ${ }^{\circledR}$ Kit Mycoplasma genitalium, the Diagenode Mycoplasma genitalium real-time PCR kit and the in house RT-PCR, respectively. The clinical specificity was 100\% for both kits and $94 \%$ for the in house RT-PCR. There was no statistically significant difference between the clinical sensitivity and specificity of these 3 methods. Moreover, there was no statistically significant difference between 